\title{
Skin/nail infections with the addition of pertuzumab to trastuzumab-based chemotherapy
}

\author{
Joanne Mortimer · Jae Jung · Yuan Yuan · Laura Kruper · Daphne Stewart • \\ Samuel Chung $\cdot$ Kim Wai Yu Mary Mendelsohn - Massimo D'Apuzzo • \\ Bernard Tegtmeier · Sanjeet Dadwal
}

Received: 30 October 2014/Accepted: 31 October 2014/Published online: 11 November 2014

(C) The Author(s) 2014. This article is published with open access at Springerlink.com

\begin{abstract}
We report a series of breast cancer patients with invasive skin and nail infections with Staphylococcus species that we attribute to the addition of pertuzumab to trastuzumab-based therapy. With the suspicion of an increased incidence of cutaneous infection in patients treated with pertuzumab and trastuzumab-based chemotherapy, treating medical oncologists identified patients receiving therapy who experienced infection. Between March and October 2014, 18 patients treated with pertuzumab and trastuzumab-based chemotherapy were found to have 21 separate skin/nail infections. Treatment was administered as neoadjuvant therapy in $12(67 \%)$ patients, adjuvant therapy in four (22\%) patients, and for metastatic disease in two $(11 \%)$ patients. Granulocyte growth factors were administered in $11(61 \%)$ patients and no patients were documented to be neutropenic. New skin and nail lesions developed as early as cycle 1 and as late as
\end{abstract}

This data will be presented at the San Antonio Breast Cancer Symposium, December 9-13, 2014.

J. Mortimer $(\bowtie) \cdot$ Y. Yuan $\cdot$ D. Stewart $\cdot$ S. Chung

Department of Medical Oncology \& Experimental Therapeutics,

City of Hope Comprehensive Cancer Center, 1500 East Duarte

Rd, Duarte, CA 91010, USA

e-mail: jmortimer@coh.org

J. Jung

Department of Dermatology, City of Hope Comprehensive

Cancer Center, 1500 East Duarte Rd, Duarte, CA 91010, USA

L. Kruper

Department of Surgery, City of Hope Comprehensive Cancer

Center, 1500 East Duarte Rd, Duarte, CA 91010, USA

K. W. Yu

Clinic Pharmacy, City of Hope Comprehensive Cancer Center,

1500 East Duarte Rd, Duarte, CA 91010, USA
8 months from initial therapy. The 21 separate infections documented were folliculitis and "bite-like" lesions (10), abscess (6), paronychia (3), and cellulitis (2). The appearance of these lesions was distinct from typical EGFR-associated skin changes. When cultures were obtained, Staphylococcus species were isolated. Quantitative immunoglobulins were assessed in $14(78 \%)$ patients and were abnormally low in six $(43 \%)$ of these patients. The skin infections resulted in treatment delay in two $(11 \%)$ patients and premature discontinuation of therapy in one patient. We believe that the skin/nail infections reported here in patients treated with the combination of pertuzumab and trastuzumab represent a previously unrecognized toxicity of adding pertuzumab to trastuzumab-based therapies.

Keywords Skin $\cdot$ Nails $\cdot$ Staphylococcus infections · Pertuzumab · Trastuzumab-based chemotherapy $\cdot$ Breast cancer

M. Mendelsohn · B. Tegtmeier

Department of Quality, Risk and Regulatory Management, City of Hope Comprehensive Cancer Center, 1500 East Duarte Rd, Duarte, CA 91010, USA

M. D’Apuzzo

Department of Pathology, City of Hope Comprehensive Cancer Center, 1500 East Duarte Rd, Duarte, CA 91010, USA

S. Dadwal

Department of Infectious Disease, City of Hope Comprehensive Cancer Center, 1500 East Duarte Rd, Duarte, CA 91010, USA 


\section{Introduction}

The HER2 protein is an important target for new drug development. Since the approval of trastuzumab in 1998, three additional HER2-directed therapies have been added to the armamentarium - lapatinib, ado-trastuzumab emtansine, and pertuzumab [1]. Lapatinib is known to produce skin and gastrointestinal toxicities that have been attributed to its dual blockade of HER2 and EGFR signaling [2]. When lapatinib is combined with trastuzumab to treat metastatic HER2-positive breast cancer, objective responses are observed even in patients who have previously been treated with trastuzumab-containing regimens. The toxicities observed with this combination are consistent with an additive effect of the two agents. Skin rashes and gastrointestinal toxicities are common, but no increase in cardiac toxicity or unanticipated side effects have been reported [3]. As second-line therapy, ado-trastuzumab emtansine results in superior disease outcomes and less toxicity than the combination of lapatinib and capecitabine [4]. Recently, the addition of pertuzumab to trastuzumab and docetaxel (PTH) has resulted in an improved objective response rate and significant improvements in both progression-free survival (PFS) and overall survival without increased toxicity $[5,6]$.

The benefit of adding pertuzumab to trastuzumab-based neoadjuvant chemotherapy was tested in the NEOSPHERE and TRYPHAENA trials. At the time of surgery, higher pathologic complete response rates were reported with the addition of pertuzumab [7, 8]. As a result, the FDA expanded the indications for pertuzumab to include both the metastatic and neoadjuvant settings. The NCCN guideline panel also recommended the addition of pertuzumab as adjuvant therapy $[9,10]$. With these expanded indications, more women with HER2-positive breast cancers are receiving treatments that combine pertuzumab and trastuzumab with chemotherapy.

Over the past 12 months, our clinical breast cancer team became aware that women treated with trastuzumab and pertuzumab were developing severe Staphylococcus skin reactions, not previously recognized in the pivotal trials or evident in the FDA briefing documents [10]. We report the natural history of what we believe is a previously unrecognized toxicity of these regimens.

\section{Methods}

In March 2014, a treating Medical Oncologist identified unusual and severe Staphylococcus infections in two patients receiving neoadjuvant PTH (patients 1 and 2). At the weekly Breast Cancer Research Conference, the cases were discussed in the context that these skin infections were potentially related to the addition of pertuzumab to the conventional regimen of a taxane, with or without carboplatin, and trastuzumab. The clinicians agreed to be attentive and report other patients who developed severe Staphylococcus skin infections. The clinical workup of these patients was not defined; however, the faculty was encouraged to take photographs of the skin lesions, and many photographs were provided by patients as they became concerned about new skin eruptions. With the identification of hypoglobulinemia in an early patient, quantitative immunoglobulins were collected on many of the subsequent patients. Antimicrobial treatment was provided on a case-by-case basis and the Departments of Infectious Disease and/or Dermatology were involved in most cases. Attribution of the infection to the combination of pertuzumab and trastuzumab was made by the Infectious Disease team.

To rule out the possibility of an institutional outbreak of Staphylococcus infections, we enlisted the help of the hospital's Infection Control team. After an extensive review, we found no increase in Staphylococcus infections since the approval of pertuzumab or since the identification of the first patient reported at City of Hope. Furthermore, some of the patients we reported were treated at different locations within the hospital, and two of the patients received therapy at a community practice site, 15 miles from the main City of Hope Duarte campus. The lot numbers for the agents administered were also documented to be different.

Approval from the IRB was obtained in order to characterize and report what appears to be a previously unrecognized toxicity of the combination of pertuzumab and trastuzumab with chemotherapy.

\section{Results}

Between March and October 2014, 18 patients with 21 separate skin/nail infections were identified. Their characteristics are summarized in Table 1 . The median age was 51 years (range 33-67 years). Twelve (67\%) patients were treated in the neoadjuvant setting, four $(22 \%)$ in the adjuvant setting, and two (11\%) for metastatic disease. Ten $(56 \%)$ patients were treated with pertuzumab, trastuzumab, carboplatin, and docetaxel (PTCH); four (22\%) with PTH; two (11\%) with pertuzumab, trastuzumab, and nab-paclitaxel (nabPT); and pertuzumab was added to maintenance trastuzumab $(\mathrm{PH})$ in the adjuvant setting for one patient. One patient (patient 12) who was initially treated with nabPT subsequently received PTCH; folliculitis developed while on PTCH. One patient (patient 2) developed three distinct infections. Of the 21 infectious episodes, seven developed after cycle 1 , four after cycle 2 , two after cycle 3 , one after cycle 4 , and seven after cycle 6 . 
Table 1 Patient Characteristics

\begin{tabular}{|c|c|c|c|c|c|c|c|}
\hline Pt. & Age & Setting & Regimen & $\begin{array}{l}\text { Cycle } \\
\text { number }\end{array}$ & Skin reaction & Organism & Total Protein/Ig levels \\
\hline 1 & 51 & Neoadjuvant & PTH & C6D12 & $\begin{array}{l}\text { Folliculitis-buttocks } \\
\text { Abscess-thumb }\end{array}$ & MSSA & $\begin{array}{l}\text { Total protein: } 4.7 \mathrm{~g} / \mathrm{dL} \\
(6.3-8.2 \mathrm{~g} / \mathrm{dL}) ; \mathrm{C} 6 \\
\text { Ig: normal; C6D28 }\end{array}$ \\
\hline \multirow[t]{3}{*}{2} & 48 & Neoadjuvant & PTH & C2D6 & Abscess-breast & - & - \\
\hline & & & & C3D6 & $\begin{array}{l}\text { Abscess-buttocks } \\
\text { and shoulder }\end{array}$ & MRSA & $\begin{array}{l}\text { Total protein: } 6.2 \mathrm{~g} / \mathrm{dL} \\
(6.3-8.2 \mathrm{~g} / \mathrm{dL}) ; \mathrm{C} 4 \mathrm{D} 10\end{array}$ \\
\hline & & & & C4-6 & Paronychia & - & $\begin{array}{l}\text { Ig: normal; C5D8 } \\
\text { IgG: } 661(700-1,600) \text {; C6D17 }\end{array}$ \\
\hline 3 & 56 & Adjuvant & PTCH & C1D17 & Folliculitis-scalp & - & $\begin{array}{l}\text { Ig: normal; C3D1, C6D34 } \\
\text { Total protein: } 6.1 \mathrm{~g} / \mathrm{dL} \\
\quad(6.3-8.2 \mathrm{~g} / \mathrm{dL}) ; \mathrm{C} 6 \mathrm{D} 23\end{array}$ \\
\hline 4 & 53 & Neoadjuvant & PTCH & C1D15 & Folliculitis-scalp & - & Ig: normal; C4D1 \\
\hline 5 & 49 & Adjuvant & PTCH & C1D20 & Folliculitis-abdomen & MRSA & $\begin{array}{l}\text { Total protein: normal } \\
\text { IgM: } 38 \text { (38-271); C3D1 }\end{array}$ \\
\hline 6 & 64 & Neoadjuvant & PTCH & C3D13 & $\begin{array}{l}\text { Abscess-shoulder } \\
\text { "Bite-like" lesions }\end{array}$ & MRSA & $\begin{array}{l}\text { Total protein: normal } \\
\text { IgG: } 707 \text { (700-1,600); C4D6 }\end{array}$ \\
\hline 7 & 47 & Adjuvant & PTCH & C1D15 & Folliculitis-scalp & - & - \\
\hline 8 & 57 & Neoadjuvant & PTH & C2D17 & Abscess-buttocks & - & - \\
\hline 9 & 46 & Neoadjuvant & PTCH & C6D15 & Paronychia-16 nails & - & $\begin{array}{l}\text { IgG: } 691 \text { (806-1,813) } \\
\text { IgM: } 51 \text { (104-522); C6D38 }\end{array}$ \\
\hline 10 & 52 & Neoadjuvant & nabPT & C1D2 & Abscess-port day 2 & MRSA & Ig: normal; C3D15 \\
\hline 11 & 51 & Metastatic & PTH & C1D2 & "Bite-like" lesions & - & IgM: 26 (38-271); C2D1 \\
\hline 12 & 33 & Metastatic & nabPT; PTCH & C12D1 & Folliculitis—arm & MSSA & Ig: normal; C12D1 \\
\hline 13 & 36 & Adjuvant & PH & C2D14 & Folliculitis-face & - & Ig: normal; C4D38 \\
\hline 14 & 50 & Neoadjuvant & PTCH & C6D25 & Paronychia & - & Ig: normal; C6D28 \\
\hline 15 & 54 & Neoadjuvant & PTCH & C2D14 & Folliculitis-scalp & - & Ig: normal; C2D15 \\
\hline 16 & 67 & Neoadjuvant & PTCH & C6D46 & Seroma with cellulitis & - & $\begin{array}{l}\text { Total protein: } 6.1 \mathrm{~g} / \mathrm{dL} \text {; C5D1 } \\
\text { IgG: } 579(700-1,600) \text {; C6D46 }\end{array}$ \\
\hline 17 & 38 & Neoadjuvant & PTCH & C1D9 & Folliculitis-face & - & - \\
\hline 18 & 56 & Neoadjuvant & nabPT & C6D65 & Cellulitis & MSSA & IgG: $556(700-1,600)$; C6D65 \\
\hline
\end{tabular}

$P T C H$ pertuzumab, trastuzumab, carboplatin, and docetaxel; $P T H$ pertuzumab, trastuzumab, and docetaxel; nabPT nab-paclitaxel, pertuzumab, and trastuzumab; PH pertuzumab and trastuzumab; MRSA methicillin-resistant Staphylococcus aureus; MSSA methicillin-sensitive Staphylococcus aureus; $C$ cycle; $D$ day; $P t$ patient

Most patients (12;67 \%) received granulocyte growth factors within $24-48 \mathrm{~h}$ of chemotherapy. No patients were documented to be neutropenic at the time of presentation with skin lesions. Cultures were performed on seven (39\%) patients; methicillin-sensitive Staphylococcus aureus (MSSA) was identified in three (43\%) of these patients and methicillin-resistant Staphylococcus aureus (MRSA) in four $(57 \%)$ of these patients. The development of skin lesions resulted in treatment delay in two $(11 \%)$ patients. Of the 12 neoadjuvant patients, eight $(67 \%)$ underwent definitive surgery and six ( $75 \%$ ) of these patients achieved a pathologic complete response.

Folliculitis involving the face or scalp was identified in six $(33 \%)$ patients with four $(22 \%)$ additional patients experiencing folliculitis and "bite-like" lesions on the arm, legs, abdomen, and buttocks. Abscesses were identified in five $(28 \%)$ patients, two of whom required incision and drainage, and one required removal of the portacath. Severe paronychial infections were seen in three $(17 \%)$ patients and one patient underwent surgical removal of two nails to facilitate healing. Two patients treated with neoadjuvant therapy developed cellulitis following their definitive local surgery. They are included in this series because of the rapid and severe nature of the infection and the documentation of hypogammaglobulinemia. Both were on maintenance trastuzumab, but had not received pertuzumab for 45 and 65 days, respectively. The clinical appearance was not typical of lapatinib-related skin rashes. Representative images of skin infections from five patients are shown in Fig. 1. 

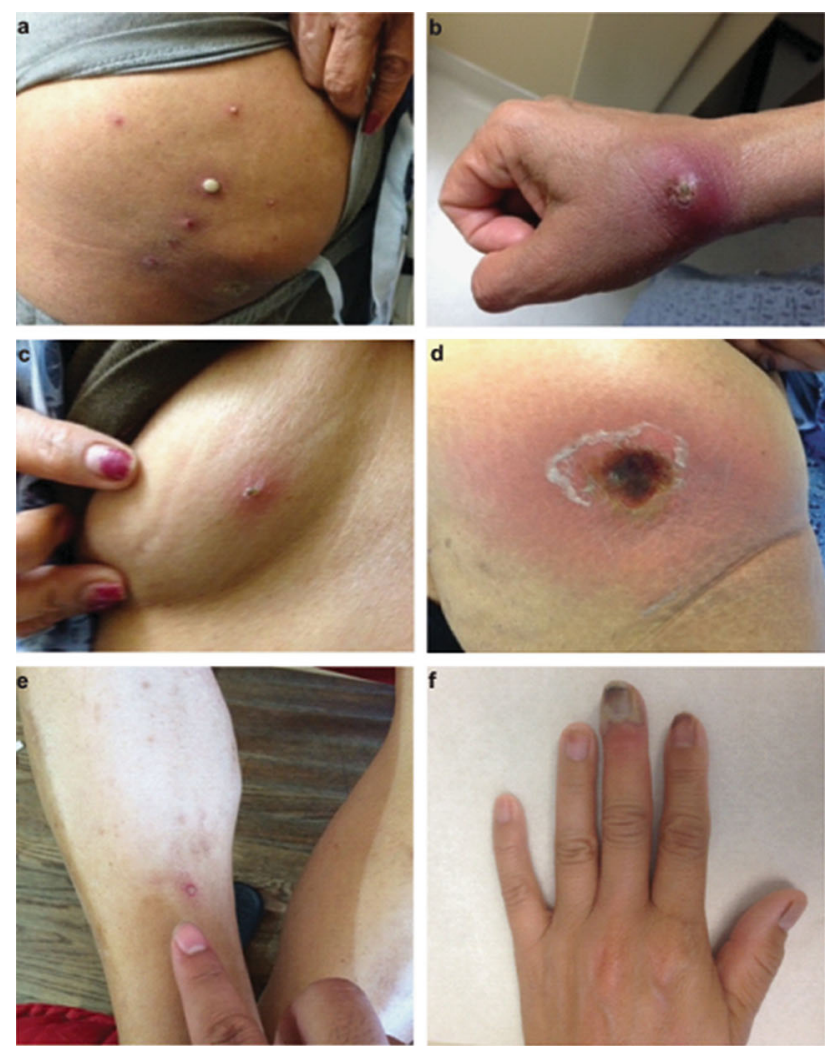

g

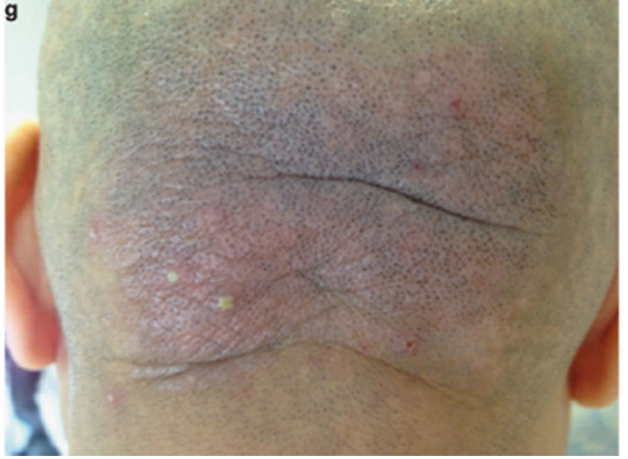

Fig. 1 Skin/nail infections a Buttocks folliculitis and $\mathbf{b}$ wrist abscess (patient 1); c Early precursor lesions and $\mathbf{d}$ buttock cellulitis (patient 2); e Early precursor lesions (patient 11); f Paronychia (patient 14); g Typical scalp folliculitis (patient 4)

Quantitative immunoglobulins were assessed in 14 (78\%) patients and were abnormally low in six (43\%) of these patients; four after cycle 6 of therapy. Multiple assessments of quantitative immunoglobulin levels were performed in three patients and became abnormal over time in all.

The following cases are representative of the unique natural history of this process.

Patient 1 is a 51-year-old East Indian woman who presented with inflammatory breast cancer involving the left breast, axilla, and supraclavicular lymph nodes. Treatment with PTH was initially well tolerated until cycle 6 , day 7 when she complained of redness of the nose and a cough. She was not cytopenic and was empirically treated with a 10-day course of cephalexin and her symptoms resolved within 3 days. On cycle 6, day 14 while on cephalexin, she complained of new skin eruptions on the breast and buttocks that looked like "insect bites." Over the subsequent 3 days these lesions increased in size, became erythematous, pus-filled, and exquisitely painful. She was seen on day 22 of cycle 6 as she was starting "maintenance trastuzumab." On that clinic visit, she received a dose of intravenous vancomycin and trimethoprim/sulfamethoxazole was initiated. Three days later, she was admitted to the hospital with worsening skin lesions. Cultures from the buttocks and wrist (Fig. 1a, b) grew Staphylococcus aureus that was sensitive to vancomycin. Skin biopsies of a new "bite" area and the abscess on the left wrist demonstrated "severe acute superficial dermatitis with interface activity, ulceration and involvement of the adnexal glands with no evidence of vasculitis." She was well-nourished; total protein was $4.7 \mathrm{~g} / \mathrm{dL}(6.3-8.2 \mathrm{~g} / \mathrm{dL})$. No further workup was performed and she was discharged on intravenous vancomycin. All lesions completely resolved. At mastectomy, $6 \mathrm{~mm}$ of residual cancer was identified in the breast; the skin and all lymph nodes were without evidence of cancer. Her postoperative course was uneventful and she continues on maintenance trastuzumab without recurrence of the skin lesions.

Patient 2 is a 48-year-old African-American woman who presented with locally advanced breast cancer on the left with pathologic confirmation of axillary node involvement. On cycle 2 , day 1 of PTCH she noted a pimple on the contralateral breast. By cycle 2, day 6 the area had increased in size (Fig. 1c). Although she was afebrile and had a normal white blood count, cephalexin was prescribed and the lesion resolved. Following cycle 4, she noted erythema and swelling on the shoulder and buttocks. She was prescribed cephalexin on day 3. By cycle 4 , day 6 both lesions had increased in size and she was admitted to the hospital for intravenous vancomycin. The shoulder lesion was incised and drained. The buttocks lesion was consistent with cellulitis (Fig. 1d) and no surgical intervention was required. Skin biopsy of the buttocks demonstrated "superficial dermatitis and no evidence of vasculitis." Cultures from the shoulder abscess grew MRSA and treatment was switched from vancomycin to daptomycin. Her total protein on admission was $6.2 \mathrm{~g} / \mathrm{dL}$ (6.3-8.2 g/dL). Both lesions resolved and she completed six cycles of chemotherapy without dose or schedule modification. After cycle 6 , she noted pain in her finger and toenails. Quantitative immunoglobulins were performed on cycle 5, day 8 and were normal. However, reassessment on cycle 6, day 17 was abnormal with IgG 661 (700-1,600).

The patient was taken to the operating room where she was found to have no evidence of tumor. The discomfort in 
Table 2 Biopsy findings

\begin{tabular}{|c|c|c|}
\hline Patient & $\begin{array}{l}\text { Site of } \\
\text { biopsy }\end{array}$ & Pathologic finding \\
\hline \multirow[t]{2}{*}{1} & Left thigh & $\begin{array}{l}\text { Vacuolar degeneration of basal layer; dense } \\
\text { mixed inflammatory infiltrate involving the } \\
\text { follicles and adnexal structures }\end{array}$ \\
\hline & $\begin{array}{l}\text { Left } \\
\text { breast }\end{array}$ & $\begin{array}{l}\text { Ulceration with vacuolar degeneration of the } \\
\text { basal layer; periadnexal abscess with } \\
\text { predominantly neutrophilic infiltrate and } \\
\text { some giant cells }\end{array}$ \\
\hline 2 & Left hip & $\begin{array}{l}\text { Hypergranulosis; compact orthokeratosis; mild } \\
\text { superficial perivascular, perineural, and } \\
\text { periadnexal infiltrates consisting of primarily } \\
\text { monocytes and lymphocytes }\end{array}$ \\
\hline 12 & $\begin{array}{l}\text { Left upper } \\
\text { arm }\end{array}$ & $\begin{array}{l}\text { Focal areas of compact keratosis and } \\
\text { parakeratosis; epidermal spongiosis; intra- } \\
\text { epidermal pustule; abscess within } \\
\text { pilosebaceous unit with dense mixed infiltrate } \\
\text { with numerous neutrophils }\end{array}$ \\
\hline 15 & Scalp & $\begin{array}{l}\text { Focal compact and orthokeratosis; vacuolar } \\
\text { degeneration of the basal layer; periadnexal } \\
\text { and perifollicular lymphocytic infiltrate with } \\
\text { sparse neutrophils }\end{array}$ \\
\hline
\end{tabular}

the finger and toenails continued to worsen postoperatively and she was able to express pus-like fluid with pressure on the nail bed. One nail spontaneously fell off; others have cracked and broken off mid-nail bed. She is tolerating maintenance trastuzumab and the nails are slowly healing.

Skin biopsies were obtained in four patients. The pathologic findings are summarized in Table 2 and representative biopsies from two patients are shown in Fig. 2. Although the clinical appearance was atypical of EGFR inhibitor rashes, the biopsy results are consistent with findings seen with other EGFR inhibitor cutaneous toxicities. However, since these results are not specific or pathognomonic, additional biopsies were not obtained in subsequent patients. The epidermis in all four biopsy specimens demonstrated areas of compact orthokeratosis and/or parakeratosis, suggesting altered keratinocyte differentiation. Additionally, the inflammation was primarily periadnexal, which is consistent with other EGFR inhibitor reactions [11, 12]. Biopsies from patient 1 showed vacuolar degeneration of the basal layer that has also been seen with acneiform reactions to EGFR inhibitors (Fig. 2a) [13].

Patient 12 presented with erythematous papules and pustules on her left arm and abdomen following her eleventh cycle of PTCH. Culture from one of the pustules revealed MSSA and she completely cleared with oral doxycycline. The lesions recurred several weeks later and biopsy of a new lesion was performed. It demonstrated focal parakeratosis with periadnexal inflammation and a hypoplastic pilosebaceous unit (Fig. 2b). Although nonspecific, these biopsy results are consistent with altered keratinocyte differentiation seen with EGFR inhibitors.

\section{Discussion}

The FDA approval of new agents is made on the basis of randomized clinical trials where the primary endpoints are tumor regression or improved PFS and overall survival. Toxicity is an important component of the approval process. However, the toxicity data are generally collected during the study conduct and are limited by the sample size. In response to patient demand, the approval process for new treatments of advanced cancers is shortening [14, 15]. As these agents become available to more patients, additional side effects become apparent. In fact, half of all serious adverse events are recognized more than seven years after FDA approval [16].

This is the first series to report the association of pertuzumab, trastuzumab, and chemotherapy with severe cutaneous staphylococcal infections. In CLEOPATRA, the
Fig. 2 Skin biopsies a highpower view of an H\&E-stained section from patient 1 showing mild acute inflammation associated with spongiosis, vacuolar degeneration, and parakeratosis; b H\&E-stained section from patient 12 showing superficial mild acute periadnexal inflammation with concurrent involvement of a hair follicle (folliculitis) and sweat glands
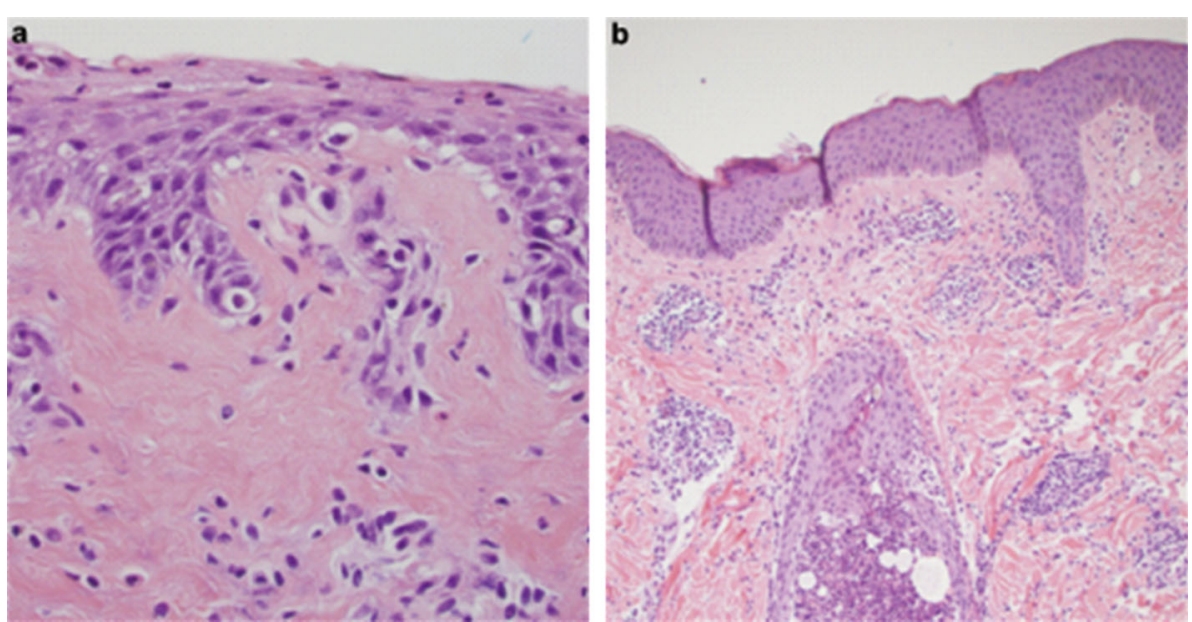
median number of cycles of docetaxel administered was eight in both arms and the frequency of all adverse effects was noted to decrease once the docetaxel was discontinued. However diarrhea, rash, dry skin, neutropenic fever, pruritus, and mucosal inflammation appeared to be more common in patients assigned to pertuzumab [6]. Nail disorders were reported in $22.9 \%$ of patients in both arms, but the incidence of grade $3 / 4$ toxicity was higher in patients receiving pertuzumab, $1.2 \%$ versus $0.3 \%$ [10]. We believe that severe skin and nail infections with Staphylococcus species are unique side effects resulting from the addition of pertuzumab to trastuzumab-based chemotherapy. This toxicity may have gone unrecognized for a number of reasons. The pertuzumab safety data are derived from three large randomized clinical trials-CLEOPATRA, NEOSPHERE, and TRYPHAENA - that enrolled patients from many institutions worldwide (Table 3). The 808 patients in CLEOPATRA were enrolled from 205 sites and 25 countries. NEOSPHERE enrolled 417 patients from 59 sites and 16 countries, and TRYPHAENA enrolled 225 patients from 44 sites and 19 countries [5, 7, 8]. It would be difficult for an investigator to appreciate a safety signal with so few patients treated at any one site.

The clinical trial designs for the randomized trials are summarized in Table 3. Three of the four arms in the NEOSPHERE trial combined trastuzumab with pertuzumab, but each administered only four cycles of therapy. All three arms of the TRYPHAENA trial utilized pertuzumab and trastuzumab, but delivered only three cycles in one arm and six cycles in two arms. In our series, folliculitis was observed early, however, the more severe infections and nail infections developed after cycles 3 and 4. In total, the toxicity data for the combination of pertuzumab and trastuzumab are based on 846 patients, 556 of whom received six cycles, 215 four cycles, and 75 three cycles. Because the addition of lapatinib to trastuzumab results in a synergistic anti-tumor effect with predictable normal tissue toxicities, synergistic toxicities from adding pertuzumab to trastuzumab were not anticipated $[3,17,18]$.

The majority of new agents are first tested in patients with metastatic disease, and it may be difficult to distinguish drug toxicity from symptoms of advanced disease. The musculoskeletal pains that occur with the aromatase inhibitors were only recognized as a toxicity of the drug class when they were used in the adjuvant setting [19]. Side effects that occur in the adjuvant versus neoadjuvant settings are less likely to be attributed to the underlying disease and safety signals are more apparent. The ongoing adjuvant APHINITY trial (Table 3) is likely to clarify new side effects related to the combined antibody therapy.

The development of pustular skin lesions may have been attributed to the steroid premedication routinely administered in patients receiving taxane-based therapies. The skin lesions described here are not typical of "steroid acne." Steroid-induced acne generally presents as monomorphic papules distributed on the upper trunk, arms and less commonly on the face [20]. Although steroid use is generally immunosuppressive, the number and severity of these reactions are unlikely to be attributed to short-course steroid use alone. Additionally, the prevalence of staphylococcal infections in this population suggests that there is a specific underlying mechanism. Although the precise pathogenesis of these reactions is unknown, we propose several overlapping and synergistic mechanisms.

Both trastuzumab and pertuzumab target HER2, an epidermal growth factor receptor overexpressed in breast cancer cells and present in keratinocytes [21]. Although these agents do not induce the classic acneiform rash seen with other EGFR inhibitors such as cetuximab and erlotinib, the combination therapy is clearly disrupting epidermal differentiation in the patients who had biopsies [13]. These results suggest that the combination of trastuzumab and pertuzumab induces a reaction that is histologically similar to other EGFR inhibitors, though clinically distinct. The difference in the morphologies of the reactions is most likely due to the variability of HER kinase expression in the skin, nails, and follicles [22, 23]. Similar to other EGFR inhibitors, this altered epidermal differentiation predisposes to infection, primarily by staphylococcal species. In one study of 221 patients on EGFR inhibitor therapy, $38 \%$ of the patients had clinical evidence of infection and over $50 \%$ of these were culture positive for staphylococci [24]. The rash and infectious disease complications with these patients were managed prophylactically with oral antibiotics (usually doxycycline or minocycline) plus a topical steroid to treat the inflammatory component. It may be beneficial to treat breast cancer patients on combined therapy with prophylactic measures as well, especially if we can determine which patients are at the highest risk of developing infectious complications. Patients who develop rash with EGFR inhibitor therapy also tend to have better outcomes [25]. It will be interesting to determine if there is a similar correlation between pertuzumab and trastuzumab treatment. The complete pathologic response rate in this small series suggests that skin/nail infections were neither a favorable nor an unfavorable predictor or response.

Recent studies have demonstrated that the immunosuppressive effect of IVIG may be related to a small percentage of glycosylated IgG-Fc present in the preparations rather than a direct effect of antigen-specific binding [26]. It may be possible that the monoclonal antibodies themselves contribute to the immunosuppression seen in these patients. We did document hypoglobulinemia in six of the patients. However, many of the affected patients had normal immunoglobulins at the time of infection and other patients became hypoglobulinemic with continuation of 
Table 3 Summary of randomized trials leading to FDA approval of pertuzumab

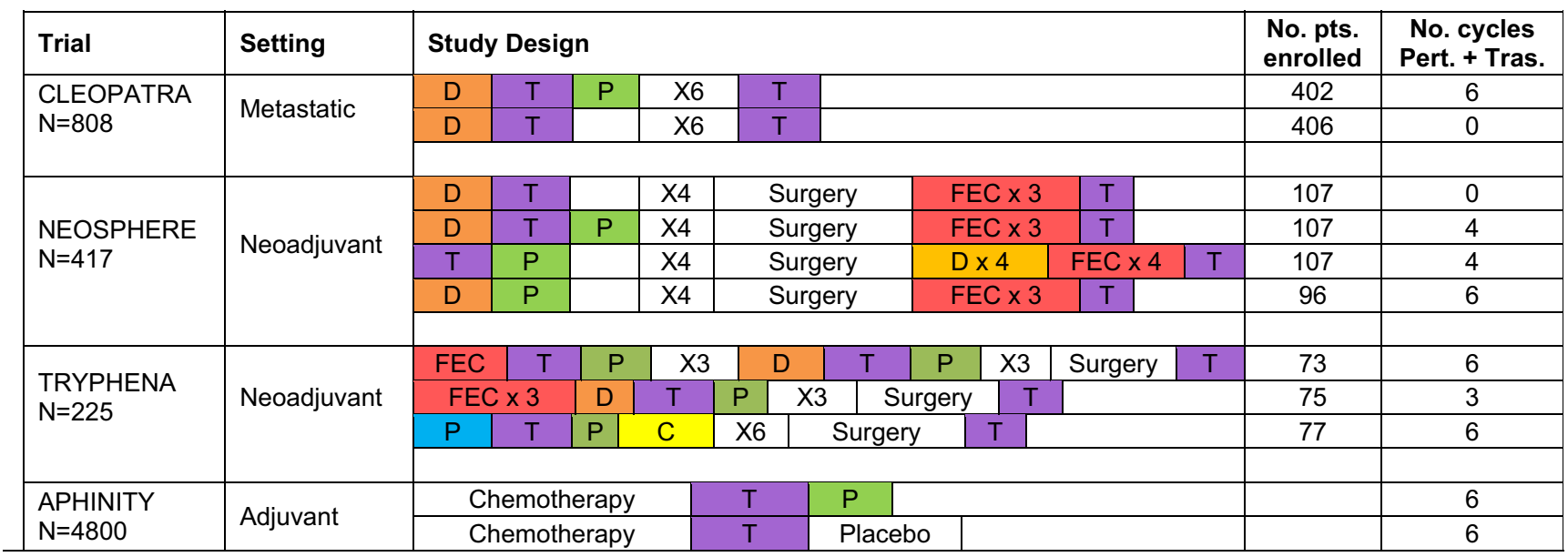

D Docetaxel Docetaxel

T Trastuzumab Trastuzumab

P Pertuzumab Pertuzumab

FEC Fluorouracil, epirubicin, cyclophosphamide Fluorouracil, epirubicin, cyclophosphamide

P Paclitaxel Paclitaxel

C Carboplatin Carboplatin

Pts Patients, No Number, Pert Pertuzumab, Tras Trastuzumab

therapy. Nonetheless, hypoglobulinemia alone does not explain the mechanism of infection.

The data presented here was derived from cases identified prospectively only after the patient developed an infection. We do not have data on the patients who did not experience any skin reaction. Amongst the group of physicians who identified the patients with skin infections reported here, the incidence was $30 \%$. To minimize any bias in assigning toxicity to pertuzumab and trastuzumab, we enlisted the help of specialists in Infectious Disease and Dermatology, and in three instances, the occurrence of a skin complication was deemed unrelated to treatment. Although this was not a prospective clinical trial, we are convinced that the occurrence of staphylococcal infections of the skin and nails is a unique toxicity observed when pertuzumab is added to trastuzumab. We are initiating a prospective trial to address cutaneous and immunologic changes in women undergoing treatment with this combined antibody therapy.

Acknowledgments The authors thank Nicola Solomon, $\mathrm{PhD}$, for assistance in editing the manuscript. Funding support for the study was provided by the National Cancer Institute (NCI) Grant \# P30 CA33572.
Conflict of interest The authors declare that they have no conflict of interest. Remuneration: Novo Nordisk (JM), BMS (DS); Consultant/advisory role: PUMA DSMB (JM); Funding: Cephalon, Novartis (JM); Stock ownership: Abbie Vie, Ariad, Bristol Meyers Squibb, Celgene, Gilead, GlaxoSmithKline, Medivation, Sanofi-Aventis, Genomic Health, Amgen, Exelixis, and Pfizer (SC).

Ethical standards Approval from the City of Hope Institutional Review Board (IRB) was obtained to conduct these studies.

Open Access This article is distributed under the terms of the Creative Commons Attribution Noncommercial License which permits any noncommercial use, distribution, and reproduction in any medium, provided the original author(s) and the source are credited.

\section{References}

1. Giordano SH, Temin S, Kirshner JJ, Chandarlapaty S, Crews JR, Davidson NE, Esteva FJ, Gonzalez-Angulo AM, Krop I, Levinson J, Lin NU, Modi S, Patt DA, Perez EA, Perlmutter J, Ramakrishna N, Winer EP (2014) Systemic therapy for patients with advanced human epidermal growth factor receptor 2-positive breast cancer: American Society of Clinical Oncology Clinical Practice Guideline. J Clin Oncol 32(19):2078-2099. doi:10.1200/ jco.2013.54.0948 
2. Geyer CE, Forster J, Lindquist D, Chan S, Romieu CG, Pienkowski T, Jagiello-Gruszfeld A, Crown J, Chan A, Kaufman B, Skarlos D, Campone M, Davidson N, Berger M, Oliva C, Rubin SD, Stein S, Cameron D (2006) Lapatinib plus capecitabine for HER2-positive advanced breast cancer. $N$ Engl J Med 355(26):2733-2743. doi:10.1056/NEJMoa064320

3. Blackwell KL, Burstein HJ, Storniolo AM, Rugo HS, Sledge G, Aktan G, Ellis C, Florance A, Vukelja S, Bischoff J, Baselga J, O'Shaughnessy J (2012) Overall survival benefit with lapatinib in combination with trastuzumab for patients with human epidermal growth factor receptor 2-positive metastatic breast cancer: final results from the EGF104900 study. J Clin Oncol 30(21):2585-2592. doi:10.1200/jco.2011.35.6725

4. Verma S, Miles D, Gianni L, Krop IE, Welslau M, Baselga J, Pegram M, Oh D-Y, Diéras V, Guardino E, Fang L, Lu MW, Olsen S, Blackwell K (2012) Trastuzumab emtansine for HER2positive advanced breast cancer. $N$ Engl $J$ Med 367(19):1783-1791. doi:10.1056/NEJMoa1209124

5. Baselga J, Cortés J, Kim S-B, Im S-A, Hegg R, Im Y-H, Roman L, Pedrini JL, Pienkowski T, Knott A, Clark E, Benyunes MC, Ross G, Swain SM (2012) Pertuzumab plus trastuzumab plus docetaxel for metastatic breast cancer. $N$ Engl $J$ Med 366(2):109-119. doi:10.1056/NEJMoa1113216

6. Swain SM, Kim S-B, Cortés J, Ro J, Semiglazov V, Campone M, Ciruelos E, Ferrero J-M, Schneeweiss A, Knott A, Clark E, Ross G, Benyunes MC, Baselga J (2013) Pertuzumab, trastuzumab, and docetaxel for HER2-positive metastatic breast cancer (CLEOPATRA study): overall survival results from a randomised, double-blind, placebo-controlled, phase 3 study. Lancet Oncol 14(6):461-471. doi:10.1016/S1470-2045(13)70130-X

7. Schneeweiss A, Chia S, Hickish T, Harvey V, Eniu A, Hegg R, Tausch C, Seo JH, Tsai Y-F, Ratnayake J, McNally V, Ross G, Cortés J (2013) Pertuzumab plus trastuzumab in combination with standard neoadjuvant anthracycline-containing and anthracycline-free chemotherapy regimens in patients with HER2positive early breast cancer: a randomized phase II cardiac safety study (TRYPHAENA). Ann Oncol 24(9):2278-2284. doi:10. 1093/annonc/mdt182

8. Gianni L, Pienkowski T, Im Y-H, Roman L, Tseng L-M, Liu M-C, Lluch A, Staroslawska E, de la Haba-Rodriguez J, Im S-A, Pedrini JL, Poirier B, Morandi P, Semiglazov V, Srimuninnimit V, Bianchi G, Szado T, Ratnayake J, Ross G, Valagussa P (2012) Efficacy and safety of neoadjuvant pertuzumab and trastuzumab in women with locally advanced, inflammatory, or early HER2positive breast cancer (NeoSphere): a randomised multicentre, open-label, phase 2 trial. Lancet Oncol 13(1):25-32. doi:10.1016/ S1470-2045(11)70336-9

9. Gradishar WJ, Anderson BO, Blair SL, Burstein HJ, Cyr A, Elias AD, Farrar WB, Forero A, Giordano SH, Goldstein LJ, Hayes DF, Hudis CA, Isakoff SJ, Ljung B-ME, Marcom PK, Mayer IA, McCormick B, Miller RS, Pegram M, Pierce LJ, Reed EC, Salerno KE, Schwartzberg LS, Smith ML, Soliman H, Somlo G, Ward JH, Wolff AC, Zellars R, Shead DA, Kumar R (2014) Breast Cancer Version 3.2014. J Natl Compr Cancer Netw 12(4):542-590

10. FDA (2013) FDA Briefing Document Oncologic Drugs Advisory Committee Meeting September 12, 2013, sBLA 125409/51 Pertuzumab (PERJETA ${ }^{\circledR}$ ); Applicant: Genentech, Inc. FDA publication: Briefing documents

11. Brodell LA, Hepper D, Lind A, Gru AA, Anadkat MJ (2013) Histopathology of acneiform eruptions in patients treated with epidermal growth factor receptor inhibitors. J Cutan Pathol 40(10):865-870. doi:10.1111/cup.12202

12. Guttman-Yassky E, Mita A, De Jonge M, Matthews L, McCarthy S, Iwata KK, Verweij J, Rowinsky EK, Krueger JG (2010)
Characterisation of the cutaneous pathology in non-small cell lung cancer (NSCLC) patients treated with the EGFR tyrosine kinase inhibitor erlotinib. Eur J Cancer 46(11):2010-2019. doi:10.1016/j.ejca.2010.04.028

13. Lacouture ME (2006) Mechanisms of cutaneous toxicities to EGFR inhibitors. Nat Rev Cancer 6(10):803-812

14. Sherman RE, Li J, Shapley S, Robb M, Woodcock J (2013) Expediting drug development: the FDA's new "breakthrough therapy" designation. N Engl J Med 369(20):1877-1880. doi:10. 1056/NEJMp1311439

15. Downing NS, Aminawung JA, Shah ND, Braunstein JB, Krumholz HM, Ross JS (2012) Regulatory review of novel therapeutics: comparison of three regulatory agencies. $\mathrm{N}$ Engl $\mathrm{J}$ Med 366(24):2284-2293. doi:10.1056/NEJMsa1200223

16. Edwards BJ, Gounder M, McKoy JM, Boyd I, Farrugia M, Migliorati C, Marx R, Ruggiero S, Dimopoulos M, Raisch DW, Singhal S, Carson K, Obadina E, Trifilio S, West D, Mehta J, Bennett CL (2008) Pharmacovigilance and reporting oversight in US FDA fast-track process: bisphosphonates and osteonecrosis of the jaw. Lancet Oncol 9(12):1166-1172

17. Baselga J, Bradbury I, Eidtmann H, Di Cosimo S, de Azambuja E, Aura C, Gómez H, Dinh P, Fauria K, Van Dooren V, Aktan G, Goldhirsch A, Chang T-W, Horváth Z, Coccia-Portugal M, Domont J, Tseng L-M, Kunz G, Sohn JH, Semiglazov V, Lerzo G, Palacova M, Probachai V, Pusztai L, Untch M, Gelber RD, Piccart-Gebhart M (2012) Lapatinib with trastuzumab for HER2positive early breast cancer (NeoALTTO): a randomised, openlabel, multicentre, phase 3 trial. Lancet 379(9816):633-640

18. Esteva FJ, Franco SX, Hagan MK, Brewster AM, Somer RA, Williams W, Florance AM, Turner S, Stein S, Perez A (2013) An open-label safety study of lapatinib plus trastuzumab plus paclitaxel in first-line HER2-positive metastatic breast cancer. Oncologist 18(6):661-666. doi:10.1634/theoncologist.2012-0129

19. Mortimer J (2010) Managing the toxicities of the aromatase inhibitors. Curr Opin Obstet Gynecol 1:56-60

20. Dessinioti C, Antoniou C, Katsambas A (2014) Acneiform eruptions. Clinics in Dermatology 32(1):24-34. doi:10.1016/j. clindermatol.2013.05.023

21. Forsberg S, Rollman O (2010) Re-epithelialization from human skin explant cultures is promoted by ligand-activated HER3 receptor. J Dermatol Sci 59(1):7-15. doi:10.1016/j.jdermsci. 2010.03.017

22. Hynes NE, Lane HA (2005) ERBB receptors and cancer: the complexity of targeted inhibitors. Nat Rev Cancer 5(5):341-354

23. Nanba D, Toki F, Barrandon Y, Higashiyama S (2013) Recent advances in the epidermal growth factor receptor/ligand system biology on skin homeostasis and keratinocyte stem cell regulation. J Dermatol Sci 72(2):81-86. doi:10.1016/j.jdermsci.2013. 05.009

24. Eilers RE, Gandhi M, Patel JD, Mulcahy MF, Agulnik M, Hensing T, Lacouture ME (2010) Dermatologic infections in cancer patients treated with epidermal growth factor receptor inhibitor therapy. J Natl Cancer Inst 102(1):47-53. doi:10.1093/jnci/ djp439

25. Perez-Soler R, Saltz L (2005) Cutaneous adverse effects with HER1/EGFR-targeted agents: Is there a silver lining? J Clin Oncol 23(22):5235-5246. doi:10.1200/jco.2005.00.6916

26. Anthony RM, Kobayashi T, Wermeling F, Ravetch JV (2011) Intravenous gammaglobulin suppresses inflammation through a novel TH2 pathway. Nature 475 (7354):110-113. doi:http:// www.nature.com/nature/journal/v475/n7354/abs/nature10134-f1. 2.html\#supplementary-information 